\title{
A case of MPO- and PR3-ANCA-negative pauci-immune renal-limited small-vessel vasculitis associated with endometrial neuroendocrine small cell carcinoma
}

\author{
Shuzo Kaneko $\cdot$ Yusuke Tsukamoto $\cdot$ \\ Kazuya Abe $\cdot$ Masayuki Yonamine • \\ Sumiko Hasegawa $\cdot$ Hiromi Hikida
}

Received: 23 October 2012/ Accepted: 24 December 2012/Published online: 26 January 2013

(C) Japanese Society of Nephrology 2013

\begin{abstract}
A 69-year-old woman was admitted to our hospital for rapid increase in serum creatinine level with microscopic hematuria and nephrotic-range proteinuria. Three months prior to admission, she became aware of atypical genital bleeding, leading her gynecologist to suspect endometrial cancer. Light microscopy examination of renal biopsy revealed crescentic glomerulonephritis with peritubular capillaritis. Immunofluorescence microscopic examination did not detect any significant staining, and no electron-dense deposits were detected by electron microscopy. No autoantibodies, including anti-myeloperoxidaseor anti-proteinase3-anti-neutrophil cytoplasmic antibodies were detected. The patient underwent total abdominal hysterectomy and bilateral salpingo-oophorectomy and was found to have endometrial neuroendocrine small cell carcinoma (NSCC), stage 1B. In her clinical course, her serum creatinine level gradually improved without immunosuppression therapy. Endometrial NSCC is a rare endometrial malignancy. This is the first case report of vasculitis associated with NSCC originating from the uterus.
\end{abstract}

Keywords MPO- and PR3-ANCA-negative . Pauci-immune - Crescentic glomerulonephritis · Peritubular capillaritis - Endometrial neuroendocrine small cell carcinoma

S. Kaneko $(\bowtie) \cdot$ Y. Tsukamoto

Department of Nephrology, Itabashi Chuo Medical Center,

2-12-7 Azusawa, Itabashiku, Tokyo 174-0051, Japan

e-mail: shuzo-kaneko10@ob.md.tsukuba.ac.jp

K. Abe $\cdot$ M. Yonamine $\cdot$ S. Hasegawa $\cdot$ H. Hikida

Department of Obstetrics and Gynecology, Itabashi

Chuo Medical Center, 2-12-7 Azusawa,

Itabashiku, Tokyo 174-0051, Japan

\section{Introduction}

Vasculitis can be associated with malignancy, most often in the form of hematological neoplasm, but sometimes as solid tumors $[1,2]$. In most cases, renal involvement in paraneoplastic vasculitis manifests as cryoglobulinemia, Henoch-Schönlein purpura and anti-neutrophil cytoplasmic antibody (ANCA)-related disease [3-5].

We describe a case of renal involvement in anti-myeloperoxidase (MPO) and anti-proteinase3 (PR3)-ANCA-negative pauci-immune small-vessel vasculitis (SVV) associated with endometrial neuroendocrine small cell carcinoma (NSCC), which is a rare endometrial malignancy. To our knowledge, this is the first case report of vasculitis associated with NSCC originating from the uterus.

\section{Case}

A 69-year-old woman was admitted to our hospital for an increase in her serum creatinine level from 82.2 to $281.1 \mu \mathrm{mol} / \mathrm{L}$ over 3 weeks. She had become aware of atypical genital bleeding 3 months prior. Her gynecologist suspected endometrial cancer based on the results of cytological examinations of the endometrium that had become available 10 days before hospitalization. Her blood pressure was $148 / 70 \mathrm{mmHg}$, height was $158 \mathrm{~cm}$, and weight was $49 \mathrm{~kg}$. No abnormalities were observed in the physical examination except for slight edema of the legs. No purpura was observed. The blood tests revealed a low level of total protein and albumin (57 and $28 \mathrm{~g} / \mathrm{L}$, respectively). Blood urea nitrogen was $8.85 \mathrm{mmol} / \mathrm{L}$, creatinine was $281.1 \mu \mathrm{mol} /$ $\mathrm{L}$ and C-reactive protein was slightly elevated at $18,900 \mu \mathrm{g} /$ L. Her hemoglobin was slightly low at $101 \mathrm{~g} / \mathrm{L}$. Levels of immunoglobulin (Ig) G, IgG subclass4, IgA and IgM were 


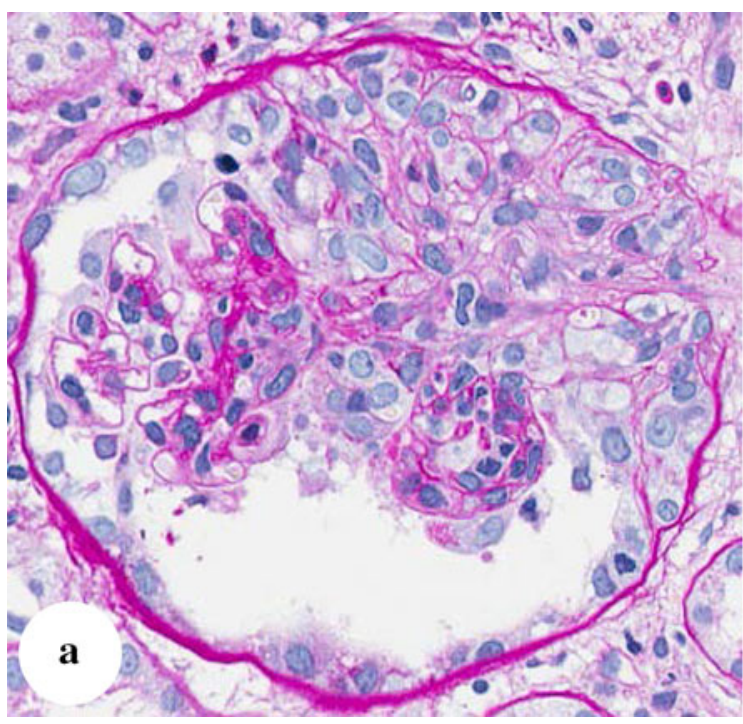

Fig. 1 Glomeruli stained with periodic acid-Schiff (a) and Masson trichrome (b) by light microscopy showing cellular crescent formation accompanied with infiltration of inflammatory cells in glomerular capillary lumen. The periodic acid-Schiff stain allowed demarcation of

all normal, at $11.06 \mathrm{~g} / \mathrm{L}, 0.296 \mathrm{~g} / \mathrm{L}, 3.17 \mathrm{~g} / \mathrm{L}$, and $0.42 \mathrm{mg} / \mathrm{d}$ 1 , respectively. Complements were all within normal limits, and she tested negative for the antinuclear antibody, MPOANCA, PR3-ANCA and anti-glomerular basement membrane antibody. Neither cryoglobulin nor monoclonal protein was detected in her blood examination. Urinalysis revealed proteinuria $(2+)$ and microscopic hematuria $(3+)$, and urine sedimentation showed a red blood cell count of over 100/HPF and white blood cell count of 10-19/HPF. The urinary protein excretion rate was in the nephrotic range at $5.0 \mathrm{~g} / 24 \mathrm{~h}$. Urine protein electrophoresis analysis showed no monoclonal protein peak. Chest X-ray showed no lung abnormality. Ultrasound imaging showed bilateral kidney swelling. Her renal biopsy was studied by light microscopy, and six of 19 total glomeruli were observed to have cellular and/or fibrocellular crescent formation occasionally accompanied by infiltrated cells in the glomerular capillary lumen (Fig. 1a, b), while the other glomeruli had only slight mesangial expansion. Extensive acute-phase tubulointerstitial damage (i.e. interstitial edema, infiltration of inflammatory cells, and tubular injury) was also observed, particularly peritubular capillaritis, with conspicuously scattered neutrophils (Fig. 2a, b). Immunofluorescence microscopic examination revealed no significant staining for $\mathrm{IgG}$, IgM, or $\mathrm{Clq}$ in either the glomerulus or tubulointerstitial area, and slight partially positive staining for IgA and $\mathrm{C} 3$ on mesangial areas or vessel walls of the vascular pole in the glomeruli (Fig. 3a-e). Infiltration of neutrophils or monocytes in the glomerular capillary lumen accompanied by swelling of the endothelial cells and foot process effacement of the podocytes was observed by electron microscopy. There were no significant electron-dense

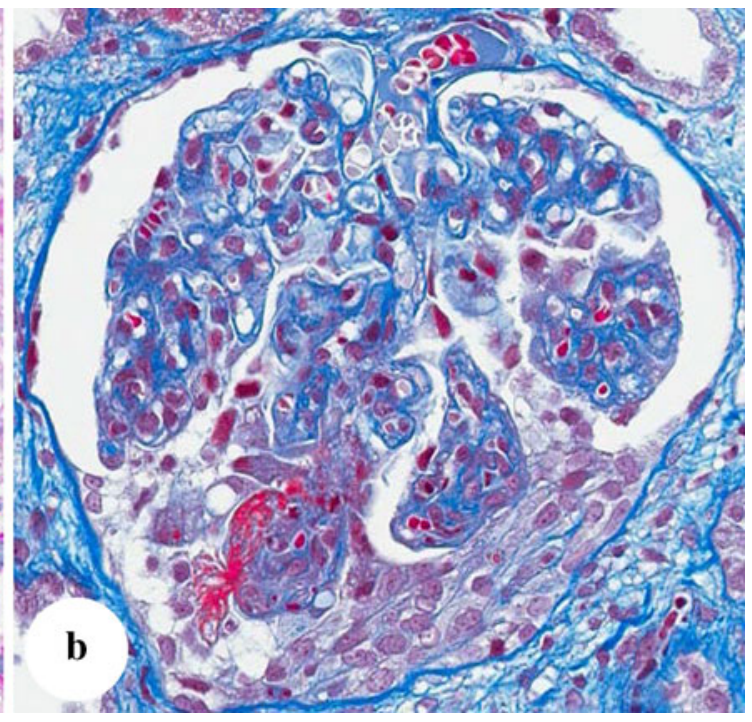

the focal dissolution of Bowman's capsule basement membrane in the direction of two to three o'clock (a). Another glomerulus stained with Masson trichrome showed a focus of segmental fibrinoid necrosis in the direction of seven o'clock, which stained red (b). a, b $\times 400$

deposits in either the glomerulus or tubular basement membrane (Fig. 4).

The patient was definitively diagnosed with endometrial NSCC based on a close examination by endometrial curettage. Our supposition that the acute nephritis had occurred in relation to the uterine malignancy was based on the close timing of the clinical onset of the diseases. We therefore decided to perform radical treatment of the malignancy prior to addressing the kidneys. Total abdominal hysterectomy and bilateral salpingo-oophorectomy were performed. Neuroendocrine differentiation was proven immunohistochemically by diffuse CD56 (i.e. neural cell adhesion molecule) expression, but not by other markers (i.e. chromogranin A or synaptophysin). The final diagnosis was endometrial NSCC, stage 1B (defined by the International Federation of Gynecology and Obstetrics). Following the surgery, her serum creatinine level gradually improved from a maximum of 291.7 to $123.7 \mu \mathrm{mol} / \mathrm{L}$ without immunosuppression therapy, but both proteinuria and microscopic hematuria persisted through her hospitalization. Just before her discharge, the urinary protein excretion rate was slightly decreased at $3.3 \mathrm{~g} / \mathrm{g}$ creatinine, while the red blood cell count of her urine sedimentation was still over 100/HPF. She was scheduled for subsequent adjuvant chemotherapy for NSCC and discharged from our hospital 38 days after her admission.

\section{Discussion}

Paraneoplastic vasculitis involves small vessels of the systemic organs, including the skin (the most common 


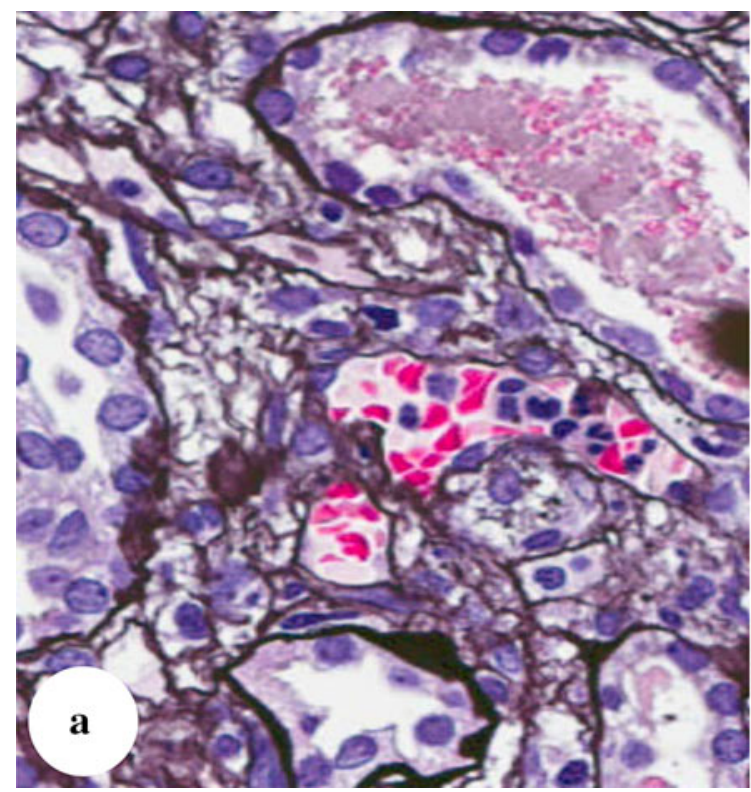

Fig. 2 Peritubular capillaritis is shown by the periodic acid-methenamine silver staining $(\mathbf{a}, \mathbf{b})$. Methenamine silver sharply stained the basement membrane of vessels and tubuli, distinguishing each structure from the interstitial area. Inflammatory cells, mainly

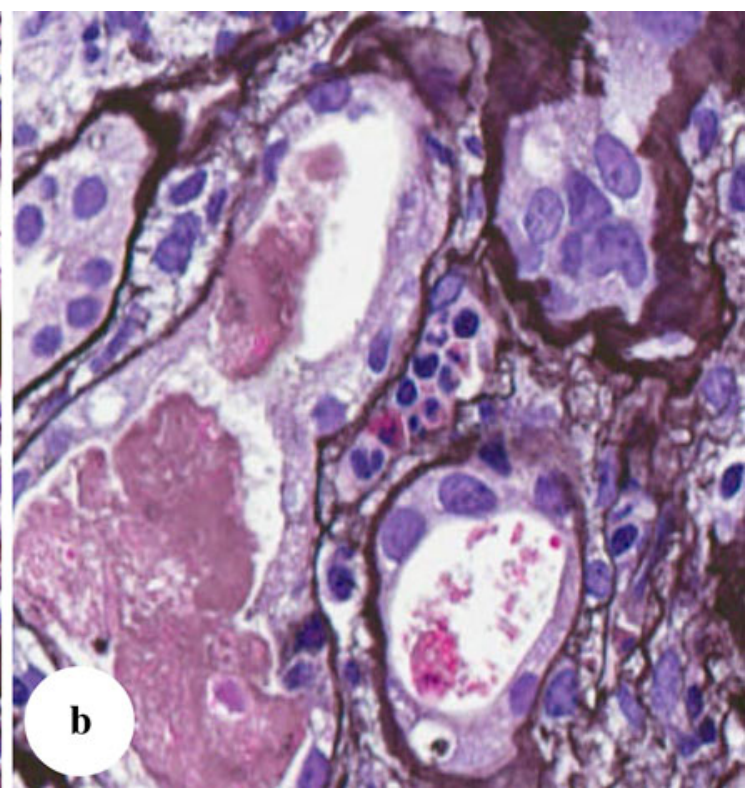

consisting of neutrophils, remained and partially marginated the lumen of the peritubular capillaries $(\mathbf{a}, \mathbf{b})$. Many red blood cells were also observed in the lumen (a). a, b $\times 400$
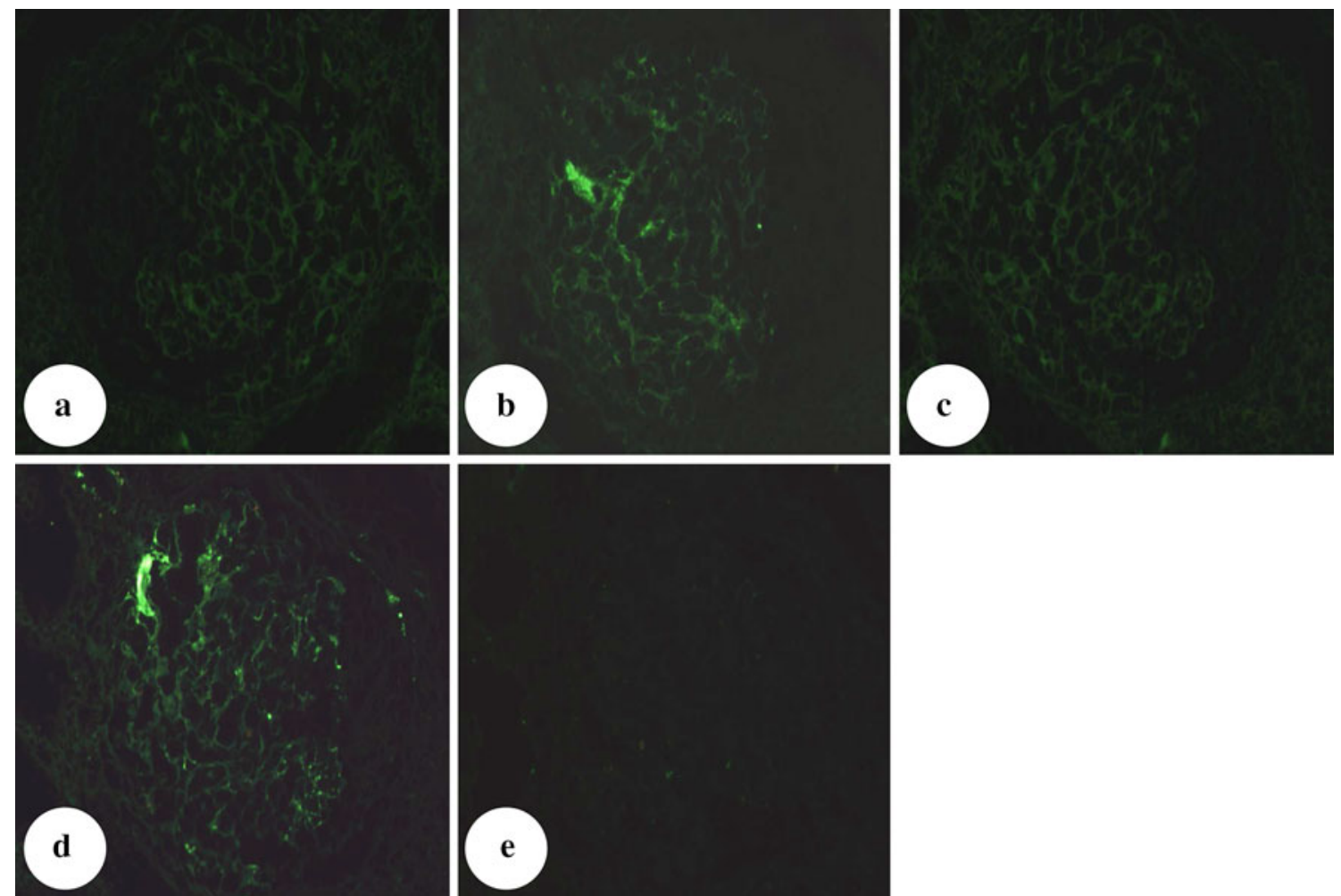

Fig. 3 Glomeruli studied by immunofluorescence microscopy are shown. Glomeruli were stained for immunoglobulin (Ig)G, IgA, IgM, $\mathrm{C} 3$ and $\mathrm{C} 1 \mathrm{q}$, respectively. No staining was observed for $\mathrm{IgG}$, IgM or

form), retina, gastrointestinal tract, lung and kidneys. In the literature, renal involvement in paraneoplastic SVV often manifests as cryoglobulinemia, Henoch-Schönlein purpura
C1q (a, c, e), while slight positive staining for $\operatorname{IgA}$ and C3 were partially observed (the former on an mesangial area, and the latter on the vessel wall of a vascular pole) $(\mathbf{b}, \mathbf{d})$

and ANCA-related disease [3-5]. The causal relation between vasculitis and malignancy is difficult to prove, but was suggested in the present case by the parallel clinical 


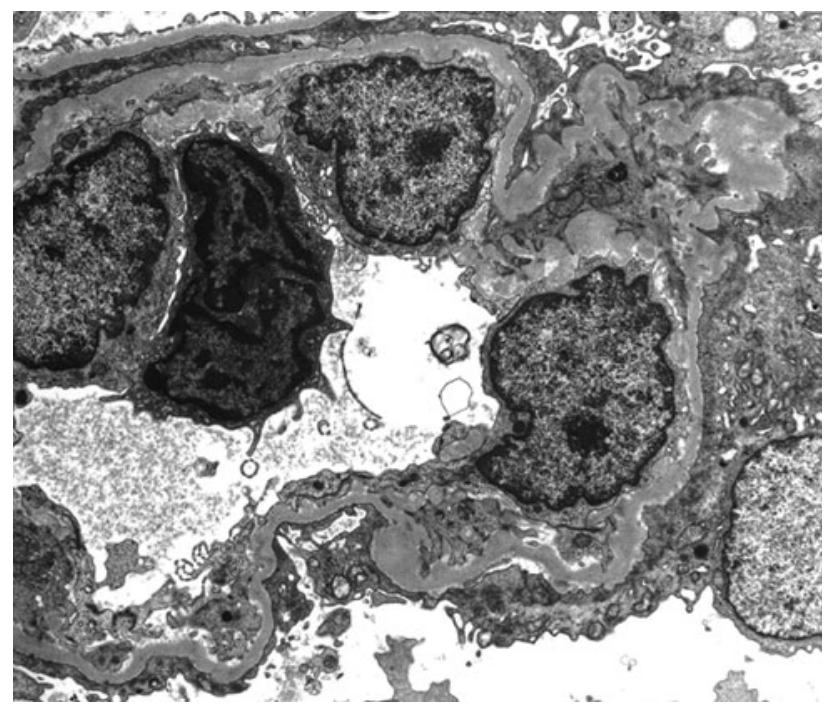

Fig. 4 An electron micrograph is shown. Adhesion of a neutrophil on endothelial cells of the glomerular capillary wall is observed. In the area of the picture, diffuse foot process effacement of podocytes and deformation (probably under destructive process) of glomerular basement membrane are observed. No significant electron-dense deposits were observed in the subendothelial area, subepithelial area or basement membrane. $\times 2500$

courses of the two pathologies. The mechanism of paraneoplastic SVV is still uncertain but is probably multifactorial, involving the development of tumor-associated antigens responsible for circulating immunocomplexes and lymphokine production by the tumor. Renal involvement in the present case of SVV showed the unusual form of MPOand PR3-ANCA-negative pauci-immune crescentic glomerulonephritis with peritubular capillaritis. There are many uncommon ANCA antigens that exist in the cytoplasm of neutrophils (or monocytes), including bacterial permeability-increasing protein, lactoferrin, lysozyme, elastase, cathepsin G, azurocidin, and lysosomal membrane protein 2 . In the case of our patient, an indirect immunofluorescence microscopy assay using alcohol-fixed neutrophils to detect ANCA was not performed. Chen and co-workers investigated ANCA-negative pauci-immune crescentic glomerulonephritisin a number of recent studies [6-9]. They reviewed the literature on its pathogenesis and found a major role of neutrophil activation in the pathway leading to neutrophil degranulation, which is accomplished by autoantibodies other than ANCAs (including antiendothelial cell antibodies [7]) or by cell-mediated immune mechanisms [i.e., interleukin (IL)-8 by neutrophil- and IL-17-producing $\mathrm{T}$ helper cells] [10-12]. In our patient, pathohistological findings showed neutrophil accumulation in peritubular capillaries, which provided one clue to the pathogenesis of the condition.

On the other hand, the present case occurred in association with endometrial NSCC, which is a rare disease (less than $1 \%$ of all endometrial cancers) [13]. Some cases of endometrial NSCC have been reported to exhibit paraneoplastic syndrome in relation to neurological or endocrinological symptoms because of its neuroendocrinological nature [14-17]. In terms of renal involvement, one case each of membranous nephropathy [18] and mesangial proliferative glomerulonephritis [19] have been reported, both of which were considered to be immunocomplex deposition disease in relation to tumor-associated antigens.

Only one case of NSCC manifesting with systemic SVV has been previously reported; that case originated from the bronchus [20]. The patient showed MPO- and PR3-ANCAnegative vasculitis involving multiple organs, including the skin, joints and kidneys, but neither neurological nor endocrinological symptoms were described. Renal involvement manifested as nephrotic syndrome and renal dysfunction, and pathohistological findings showed pauci-immune focal segmental proliferative glomerulonephritis with cellular crescents.

The specific neuroendocrinological nature of NSCC may not be directly related to the pathogenesis and development of vasculitis because neither neurological nor endocrinological symptoms were manifested in either our case or in the case originating from the bronchus.

In summary, we report a case of MPO- and PR3-ANCAnegative pauci-immune renal-limited SVV associated with endometrial NSCC. Based on this case, we hypothesize that cytokine/lymphokine production introduced by the tumor may have promoted neutrophil priming (i.e. the surfacing of cytoplasmic antigens or Fc receptors) or antibody production, leading to the outbreak and development of SVV, similar to what occurs in both ANCA- and non-ANCA-associated vasculitis. Further investigation of this mechanism is needed.

\section{References}

1. Naschitz JE, Yeshurun D, Eldar S, et al. Diagnosis of cancerassociated disorders. Cancer. 1996;77:1759-67.

2. Hutson TE, Hoffman GS. Temporal concurrence of vasculitis and cancer: a report of 12 cases. Arthritis Care Res. 2000;13:417-23.

3. Molina-Garrido MJ, Guillén-Ponce C. A revision on cryoglobulinaemia associated to neoplastic diseases. Clin Transl Oncol. 2007;9:229-36.

4. Pankhurst T, Savage CO, Gordon C, Harper L. Malignancy is increased in ANCA-associated vasculitis. Rheumatology (Oxford). 2004;43:1532-5.

5. Pertuiset E, Lioté F, Launay-Russ E, et al. Adult HenochSchonlein purpura associated with malignancy. Semin Arthr Rheum. 2000;29:360-7.

6. Chen M, Kallenberg CG, Zhao MH. ANCA-negative pauciimmune crescentic glomerulonephritis. Nat Rev Nephrol. 2009; 5:313-8.

7. Cong M, Chen M, Zhang JJ, Hu Z, Zhao MH. Anti-endothelial cell antibodies in antineutrophil cytoplasmic antibodies negative 
pauci-immune crescentic glomerulonephritis. Nephrology (Carlton). 2008;13:228-34.

8. Wang $\mathrm{F}$, Chen $\mathrm{M}$, Zhao $\mathrm{MH}$. Neutrophil degranulation in antineutrophil cytoplasmic antibody-negative pauci-immune crescentic glomerulonephritis. J Nephrol. 2009;22:491-6.

9. Xing GQ, Chen M, Liu G, Zheng XEJ, Zhao MH. Differential deposition of $\mathrm{C} 4 \mathrm{~d}$ and MBL in glomeruli of patients with ANCAnegative pauci-immune crescentic glomerulonephritis. J Clin Immunol. 2010;30:144-56.

10. Cockwell P, Brooks CJ, Adu D, Savage CO. Interleukin-8: a pathogenetic role in antineutrophil cytoplasmic autoantibodyassociated glomerulonephritis. Kidney Int. 1999;55:852-63.

11. Abdulahad WH, Stegeman CA, Limburg PC, Kallenberg CG. Skewed distribution of $\mathrm{T}_{\mathrm{H}} 17$ lymphocytes in patients with Wegener's granulomatosis in remission. Arthr Rheum. 2008;58: 2196-205.

12. Laan M, Cui ZH, Hoshino $H$, et al. Neutrophil recruitment by human IL-17 via C-X-C chemokine release in the airways. J Immunol. 1999;162:2347-52.

13. Huntsman DG, Clement PB, Gilks CB, Scully RE. Small cell carcinoma of the endometrium: a clinicopathological study of sixteen cases. Am J Surg Pathol. 1994;18:364-75.

14. Ju W, Park IA, Kim SH, Lee SE, Kim SC. Small cell carcinoma of the uterine corpus manifesting with visual dysfunction. Gynecol Oncol. 2005;99:504-6.
15. Sekiguchi I, Suzuki M, Sato I, Ohkawa T, Kawashima H, Tsuchida S. Rare case of small-cell carcinoma arising from the endometrium with paraneoplastic retinopathy. Gynecol Oncol. 1998;71:454-7.

16. Camp E, Brunier MN, Merino MJ. Small cell carcinoma of the endometrium with associated ocular paraneoplastic syndrome. Cancer. 1992;69:2283-8.

17. Sato H, Kanai G, Kajiwara H, Itoh J, Osamura RY. Small-cell carcinoma of the endometrium presenting as Cusing's syndrome. Endocr J. 2010;57:31-8.

18. Meydanli MM, Erguvan R, Altinok MT, Ataoglu O, Kafkasli A. Rare case of neuroendocrine small cell carcinoma of the endometrium with paraneoplastic membranous glomerulonephritis. Tumori. 2003;89:213-7.

19. Jermanovich NB, Giammarco R, Ginsberg SJ, Tinsley RW, Jones DB. Small cell anaplastic carcinoma of the lung with mesangial proliferative glomerulonephritis. Arch Intern Med. 1982;142: 397-9.

20. Ponge T, Boutoille D, Moreau A, et al. Systemic vasculitis in a patient with small-cell neuroendocrine bronchial cancer. Eur Respir J. 1998;12:1228-9. 